\title{
Rapid, Activation-Induced Redistribution of Ionotropic Glutamate Receptors in Cultured Hippocampal Neurons
}

\author{
Dmitri V. Lissin, ${ }^{1}$ Reed C. Carroll,, ${ }^{1}$ Roger A. Nicoll, ${ }^{2,3}$ Robert C. Malenka, ${ }^{1,3}$ and Mark von Zastrow ${ }^{1,2}$ \\ Departments of ${ }^{1}$ Psychiatry, ${ }^{2}$ Cellular and Molecular Pharmacology, and ${ }^{3}$ Physiology, University of California at San \\ Francisco, San Francisco, California 94143
}

\begin{abstract}
We have examined the membrane localization of an AMPA receptor subunit (GluR1) and an NMDA receptor subunit (NR1) endogenously expressed in primary cultures of rat hippocampal neurons. In unstimulated cultures, both GluR1 and NR1 subunits were concentrated in SV2-positive synaptic clusters associated with dendritic shafts and spines. Within $5 \mathrm{~min}$ after the addition of $100 \mu \mathrm{M}$ glutamate to the culture medium, a rapid and selective redistribution of GluR1 subunits away from a subset of synaptic sites was observed. This redistribution of GluR1 subunits was also induced by AMPA, did not require NMDA receptor activation, did not result from ligand-induced
\end{abstract}

AMPA- and NMDA-type glutamate receptors are ligand-gated ion channels that play a critical role in mediating excitatory neurotransmission and synaptic plasticity. It is well established that AMPA and NMDA receptors are concentrated in the postsynaptic membrane, but immunocytochemical and biochemical studies suggest that this may occur via distinct molecular mechanisms (Dong et al., 1997; Kornau et al., 1997; Sheng, 1997; O'Brien et al., 1998). Furthermore, recent studies suggest that the distribution of AMPA (Lissin et al., 1998; Turrigiano et al., 1998) and NMDA (Rao and Craig, 1997) receptors in the postsynaptic plasma membrane are regulated by synaptic activity, a regulation that may play an important role in certain forms of synaptic plasticity. Previously, we (Lissin et al., 1998) and others (Rao and Craig, 1997) have used immunocytochemical techniques to demonstrate subtype-selective, activity-dependent changes in the number of glutamate receptors associated with individual synapses in cultured neurons. To date, these studies have detected redistribution of glutamate receptors occurring only over a prolonged time scale, ranging from several days to weeks for detectable changes in receptor number at synapses to be observed. Physiological studies, on the other hand, suggest that the number of functional AMPA receptors at the synapse can be regulated much more rapidly (Isaac et al., 1995; Liao et al., 1995; Durand et al., 1996). However, the functional status of glutamate receptors can be regulated by posttranslational modifications, such as phos-

Received July 22, 1998; revised Nov. 13, 1998; accepted Nov. 25, 1998.

These studies were supported by grants from the National Institutes of Health (to R.C.M., R.A.N., and M.v.Z.), the Human Frontier Science Program (to R.C.M.), and the McKnight Endowment Fund for Neuroscience (to R.C.M.). R.C.C. was supported by a National Research Service Award from the National Institutes of Health. We thank Chris Billante and Stephen Gomperts for preparing hippocampal cultures, Richard Huganir and Peter Sargent for providing antibodies, and Heather W. Deacon for expert advice and assistance with confocal microscopy.

Correspondence should be addressed to Mark von Zastrow, University of California at San Francisco, Box 0984IRE/UCSF, Room LP-A104 LPPI, 401 Parnassus Avenue, San Francisco, CA 94143-0984.

Copyright (C) 1999 Society for Neuroscience $\quad 0270-6474 / 99 / 191263-10 \$ 05.00 / 0$ neurotoxicity, and was reversible after the removal of agonist. The activation-induced redistribution of GluR1 subunits was associated with a pronounced $(\sim 50 \%)$ decrease in the frequency of miniature EPSCs, consistent with a role of GluR1 subunit redistribution in mediating rapid regulation of synaptic efficacy. We conclude that ionotropic glutamate receptors are regulated in native neurons by rapid, subtype-specific membrane trafficking, which may modulate synaptic transmission in response to physiological or pathophysiological activation.

Key words: glutamate; synaptic transmission; synaptic plasticity; receptor regulation; redistribution; immunocytochemistry

phorylation, that do not require physical movement of the receptor protein. Therefore, whether or not glutamate receptors actually can undergo rapid physical redistribution in neurons is an important question that has not been directly addressed previously.

In the present study, we have used immunocytochemical methods to visualize the localization of endogenously expressed GluR1 and NR1, subunits of AMPA and NMDA receptors, respectively, in cultured hippocampal neurons. Because ligand activation has been found to cause rapid internalization of many other types of signal-transducing receptors, including receptor tyrosine kinases and G-protein-coupled receptors (Hausdorff et al., 1990; Vieira et al., 1996; Grady et al., 1997), we asked whether an analogous mechanism might apply to ionotropic glutamate receptors. Within several minutes after ligand-induced activation, we find that GluR1-containing receptors associated with synapses redistribute away from these structures and subsequently accumulate in intracellular membranes located within dendritic shafts and cell bodies of stimulated neurons. This process affects GluR1-containing, but not NR1-containing, receptors, is reversible, and is not initiated by membrane depolarization alone, suggesting that it may be mediated directly by ligand-induced activation of AMPA receptors. Furthermore, this rapid relocalization of AMPA receptors is associated with a significant change in synaptic transmission, suggesting that the rapid redistribution of GluR1-containing receptors may play an important role in modulating synaptic efficacy.

\section{MATERIALS AND METHODS}

Cultured hippocampal neurons. Hippocampi of newborn (postnatal day 0) Sprague Dawley rat pups were removed, and the dentate gyri were grossly dissected. Cells derived from the remaining tissue were plated as described previously (Lester et al., 1989), except that papain was not used. B27-supplemented (Life Technologies, Grand Island, NY) primary hippocampal cultures were prepared as described previously (Brewer et al., 1993), and $50 \mathrm{U} / \mathrm{ml}$ streptomycin and penicillin were added. One-half 
of the growth medium was exchanged $1 \mathrm{~d}$ after plating and weekly thereafter.

Immunocytochemistry. Cells were fixed with methanol for $10 \mathrm{~min}$ at $-20^{\circ} \mathrm{C}$ and permeabilized with $0.1 \%$ Triton $\mathrm{X}-100$ in PBS. AMPA receptors were detected using rabbit polyclonal anti-GluR1 [recognizing the C-terminal cytoplasmic domain, a generous gift from Dr. Richard Huganir (Dong et al., 1997)]. NMDA receptors were detected using mouse monoclonal anti-NMDAR1 (PharMingen, San Diego, CA) antibodies or rabbit polyclonal anti-NMDAR1 [recognizing the C-terminal cytoplasmic domain, a generous gift from Dr. Richard Huganir (Johns Hopkins University, Baltimore, MD) (Dong et al., 1997)]. Synapses were labeled using either rabbit anti-synaptophysin (PharMingen) or mouse anti-SV2 (a generous gift from Dr. Peter Sargent, University of California, San Francisco, CA), depending on the species of the anti-receptor antibody used. SV2 and synaptophysin immunoreactivity colocalized extensively in our cultures, so these antibodies were used interchangeably as synaptic markers. Fluorochrome-conjugated anti-mouse and antirabbit secondary antibodies were obtained from Jackson ImmunoResearch (West Grove, PA). Identification of glutamate receptor clusters and their colocalization with synaptic markers was accomplished with dual color microscopy using a Nikon $60 \times$ objective (NA 1.4) and standard fluorescein and $\mathrm{Cy} 3$ filter sets (Omega). Fluorescent images were acquired using a cooled digital CCD camera (Princeton Instruments, Inc.). Minimal bleed-through between channels was confirmed by imaging single-labeled specimens. Twelve-bit images were written linearly to an eight-bit data set after normalizing images to maximize usable contrast range using IPLab Spectrum software (Signal Analytics). For display in figures, monochrome and merged color images were processed using Adobe Photoshop software (Adobe Systems, San Jose, CA). For quantitative analysis, raw data present in the normalized (but unprocessed) eight-bit image were analyzed in a blinded manner. Synaptic structures (identified as synaptophysin- or SV2-positive puncta) were scored as glutamate receptor-positive if the intensity of receptor immunoreactivity was more than twofold higher than the background level in the unprocessed image. Using this criterion, receptor-negative synaptic sites had uniformly low levels of receptor immunoreactivity [mean \pm SD intensity of $27 \pm 20$ and $19 \pm 18$ in a representative eight-bit (256 gray scale) image of GluR1 and NR1 immunoreactivity, respectively]. In contrast, puncta scored as receptor-positive by this criterion were typically more than fivefold higher in their intensity of immunochemical staining $(157 \pm 60$ and $131 \pm 55$ for GluR1 and NMDAR1 immunoreactivity, respectively, in the same unprocessed eight-bit images). Fluorescein-conjugated phalloidin (Molecular Probes, Eugene, OR) was used to label actin enriched in dendritic spines. Colocalization of receptors to phalloidin-positive structures was determined by costaining with rabbit anti-GluR1, followed by Cy3-conjugated secondary antibody. Epifluorescence imaging of these specimens was performed as described above. Confocal microscopy was performed using a Leica (Nussloch, Germany) TCS NT confocal microscope and a Leica $100 \times$ (NA 1.4) objective, using dual laser excitation to minimize bleed-through between FITC and Cy3 channels and collecting serial optical sections sampled at $0.5 \mu \mathrm{m}$ steps. Three-dimensional image reconstruction was performed using Leica TCS Image software. "End-on" views of dendritic processes were generated from dual label data sets by calculating a maximum projection image, representing the maximum of all intensity values along the direction of the $z^{\prime}$-axis.

Electrophysiology in cultured hippocampal neurons. Cultures (12- to 15-d-old) were prepared exactly as for the immunocytochemistry experiments. Whole-cell recordings were made with an Axopatch-1D amplifier using low resistance patch pipettes (2-5 M 2 ). Pipette solutions contained (in $\mathrm{mm}$ ): $116 \mathrm{~K}$-gluconate, $6 \mathrm{KCl}, 2 \mathrm{NaCl}, 20 \mathrm{HEPES}, 0.5 \mathrm{EGTA}, 2$ $\mathrm{MgATP}$, and $0.3 \mathrm{NaGTP}$, adjusted to $\mathrm{pH} 7.2$ with KOH. The extracellular solution contained (in $\mathrm{mM}$ ): $115 \mathrm{NaCl}, 5 \mathrm{KCl}, 5 \mathrm{HEPES}, 20$ glucose, $1.8 \mathrm{CaCl}_{2}$, and $1 \mathrm{MgCl}_{2}$, adjusted to $\mathrm{pH} 7.3$ with $\mathrm{NaOH}$ (maintained at $35^{\circ} \mathrm{C}$ ). For recording miniature EPSCs (mEPSCs), cells were held at -60 $\mathrm{mV}$ in extracellular solution containing $10 \mathrm{~mm}$ lidocaine. mEPSCs were acquired for $10 \mathrm{~min}$, after which the lidocaine was washed out and cells were placed in current clamp during the addition of AMPA. After a 15 min treatment with AMPA, lidocaine was added back to the extracellular solution, and the cells were voltage clamped at $-60 \mathrm{mV}$ for further acquisition. mEPSCs were acquired using Fetchex (Axon Instruments, Foster City, CA) and were analyzed using MiniTM (J. Steinbach, Washington University, St. Louis, MO). Threshold mEPSC amplitude was set at 3 pA, and 300-1000 events were collected and averaged to calculate the mean mEPSC amplitude and frequency for each culture preparation examined.

\section{RESULTS}

The localization of AMPA and NMDA receptors in primary cultures of rat hippocampal neurons was examined using antibodies recognizing endogenous GluR1 (an AMPA receptor subunit) and NR1 (an NMDA receptor subunit). All studies were performed in 2-week-old cultures in which glutamate receptors have been demonstrated previously to be localized at postsynaptic sites in dendritic spines (Craig et al., 1993; O’Brien et al., 1998).

Accordingly, in control cultures, GluR1 subunits (detected using an antibody recognizing the C-terminal cytoplasmic domain) were visualized in numerous puncta associated with synaptic sites identified by SV2 immunoreactivity (Fig. 1 $A$, left panels, arrow, example of a colocalized synaptic puncta). Quantitation of this colocalization indicated that, in control cultures, the vast majority $(\sim 85 \%)$ of GluR1-containing puncta colocalized with synapses (Fig. 1C, filled bar), and most synapses were associated with detectable GluR1 immunoreactivity (Fig. 1D, filled bar). To determine whether ligand-induced activation causes a physical redistribution of glutamate receptors, as is the case for certain other classes of signal-transducing receptors [such as G-protein-coupled receptors and receptor tyrosine kinases (Vieira et al., 1996; Grady et al., 1997)], we applied $100 \mu \mathrm{M}$ glutamate and then examined the localization of glutamate receptor subunits. This manipulation caused a rapid and pronounced redistribution of GluR1-containing receptors, as indicated by visual inspection of fluorescence micrographs (Fig. $1 B$, arrows, example of a GluR1-negative synapse). Quantitation of these observations, visualized in multiple fields $(n=20$ fields acquired blindly from a total of three separate experiments), confirmed a substantial decrease in the proportion of GluR1positive puncta associated with synapses (Fig. 1C, open bar) occurring within $15 \mathrm{~min}$ after application of glutamate and a concomitant decrease in the number of synapses containing detectable GluR1 immunoreactivity (Fig. 1D, open bar). This glutamate-induced redistribution of GluR1 subunits was not associated with any detectable change in the number of SV2 puncta visualized in the cultured neurons (data not shown), indicating that glutamate caused a rapid redistribution of GluR1 subunits from synaptic to nonsynaptic sites without changing the overall number of synapses.

In contrast to the rapid redistribution of GluR1 immunoreactivity observed in the presence of glutamate, no apparent change in the localization of NR1 subunits (detected using a rabbit antibody recognizing the $\mathrm{C}$-terminal cytoplasmic domain) was observed under the same conditions (Fig. $2 A, B$ ). Quantitation of these observations in the unprocessed images (collected from 20 fields acquired blindly from a total of three separate experiments) confirmed the absence of any detectable change in the synaptic localization of NR1 subunits (Fig. 2C,D). Similarly, no apparent change in the synaptic localization of NR1 subunits was detected using a monoclonal antibody recognizing a different epitope of NR1 compared with the alternate synaptic marker synaptophysin (data not shown). To further confirm the subunit specificity of this receptor redistribution, the localization of NR1 and GluR1 was examined simultaneously in the same neurons, using rabbit anti-GluR1 and mouse anti-NR1 antibodies. Clusters of GluR1 and NR1 immunoreactivity were extensively colocalized in unstimulated cultures, consistent with the localization of both AMPA and NMDA receptors in the same postsynaptic mem- 


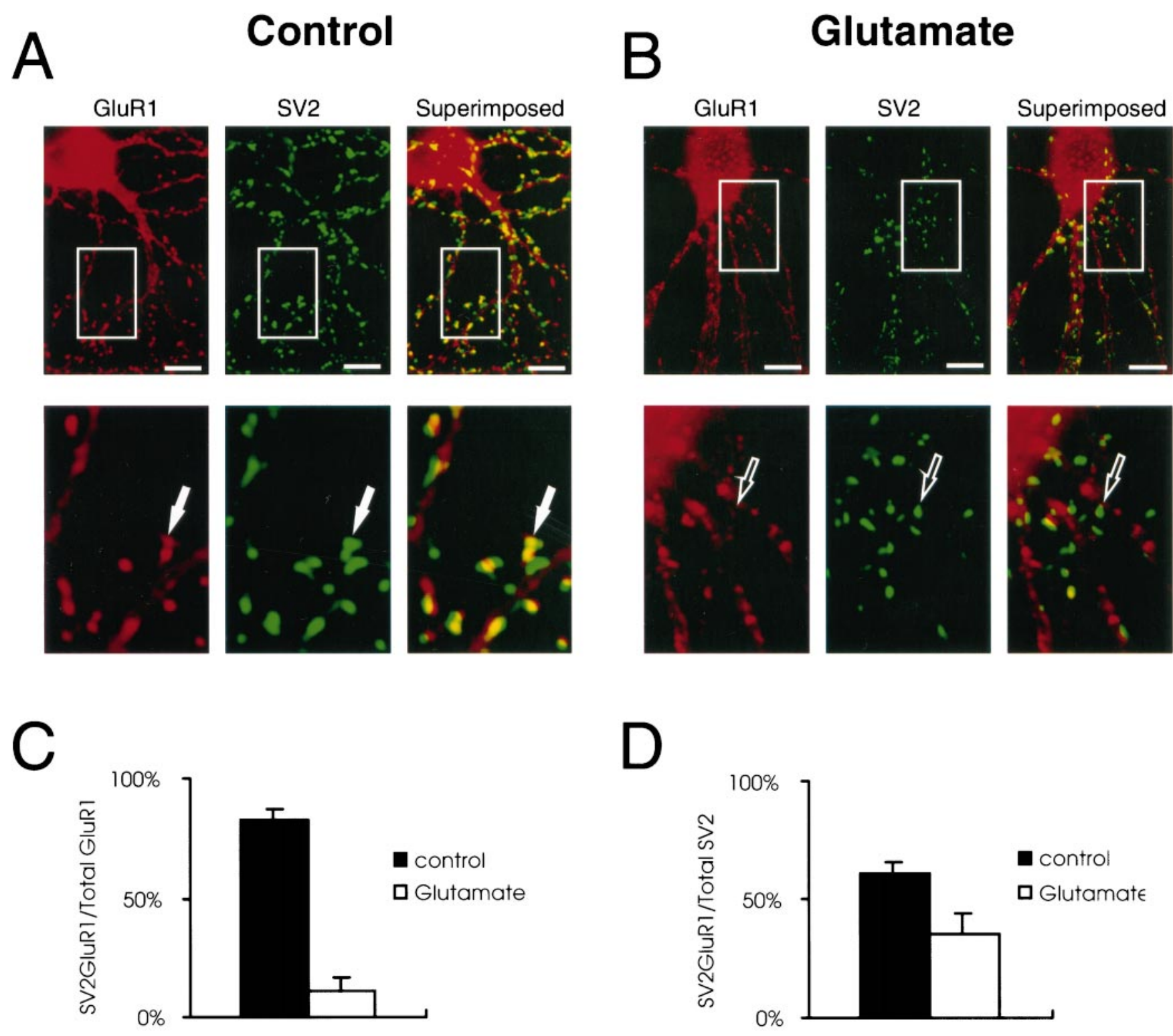

Figure 1. Glutamate causes a rapid redistribution of GluR1 subunits away from synapses in cultured hippocampal neurons. $A$, Immunocytochemical localization of GluR1 and SV2 in cultured hippocampal neurons incubated in the absence of added glutamate. A higher magnification image of the region indicated by the box is displayed in the bottom panels. Arrows in these panels point out an example of a GluR1 puncta colocalized with SV2 immunoreactivity. $B$, Immunocytochemical localization of GluR1 subunits and SV2 in neurons incubated in the presence of $100 \mu \mathrm{M}$ glutamate for 15 min. Scale bars, $5 \mu \mathrm{m}$. C, Quantitation of the proportion of GluR1-immunoreactive puncta associated with SV2 in control (untreated) and glutamate-treated neurons. D, Quantitation of the proportion of SV2-positive synaptic puncta associated with detectable concentrations of GluR1 immunoreactivity. Data are derived from analysis of a total of 20 independent fields, selected at random and examined in a blinded manner, from three separate experiments. GluR1-positive puncta were defined by a GluR1 immunoreactivity at least twofold greater than background (unclustered regions) in the raw (unprocessed) fluorescence image, as described in Materials and Methods. Bars represent the mean proportion, and error bars represent the SEM from the independent fields $(n=20)$.

branes (Fig. 3A, arrows, examples of colocalized puncta). After the application of glutamate, however, the localization of GluR1 relative to NR1 subunits changed rapidly. This was indicated by the appearance of an increased proportion of puncta selectively containing only GluR1 or NR1 immunoreactivity, but not both, in cultures incubated in the presence of glutamate for $30 \mathrm{~min}$ (Fig. $3 B$, arrows, examples of puncta associated with high levels of NR1 immunoreactivity but devoid of detectable GluR1 immunoreactivity; similar results were observed in four independent experiments).

The selective agonist AMPA also caused a rapid and pro- nounced redistribution of GluR1 subunits (Fig. 4A,B). Redistribution of GluR1 could be observed as early as 5 min after application of AMPA to the cultures, although the maximum effect appeared to require 10-15 min incubation in the continuous presence of AMPA. Significantly, this redistribution of GluR1 was observed when AMPA was applied in the presence of high concentrations of the selective NMDA antagonist 2-amino5-phosphonovaleric acid (D-APV) $(100 \mu \mathrm{M})$, indicating that this redistribution was independent of NMDA receptor activation as a consequence of AMPA-induced neuronal activity (Fig. 4C). To determine whether this effect was a consequence of the mem- 
A
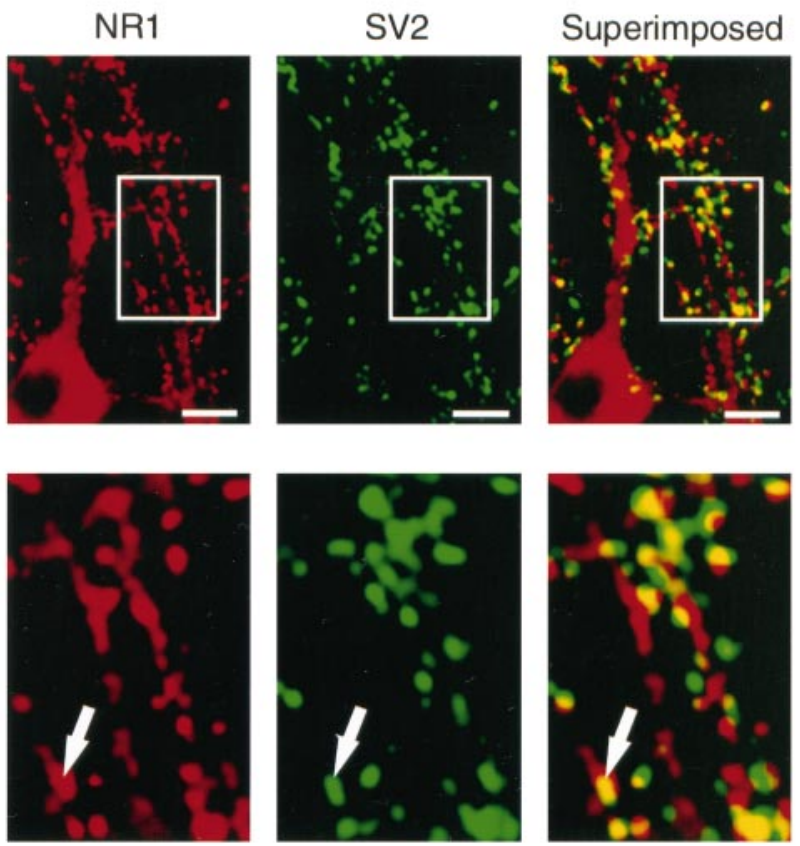

\section{Glutamate}
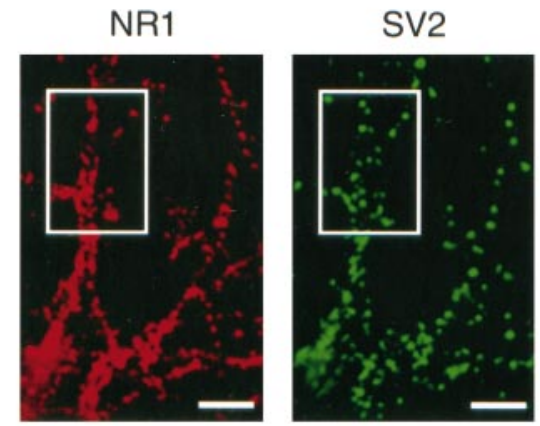

Superimposed
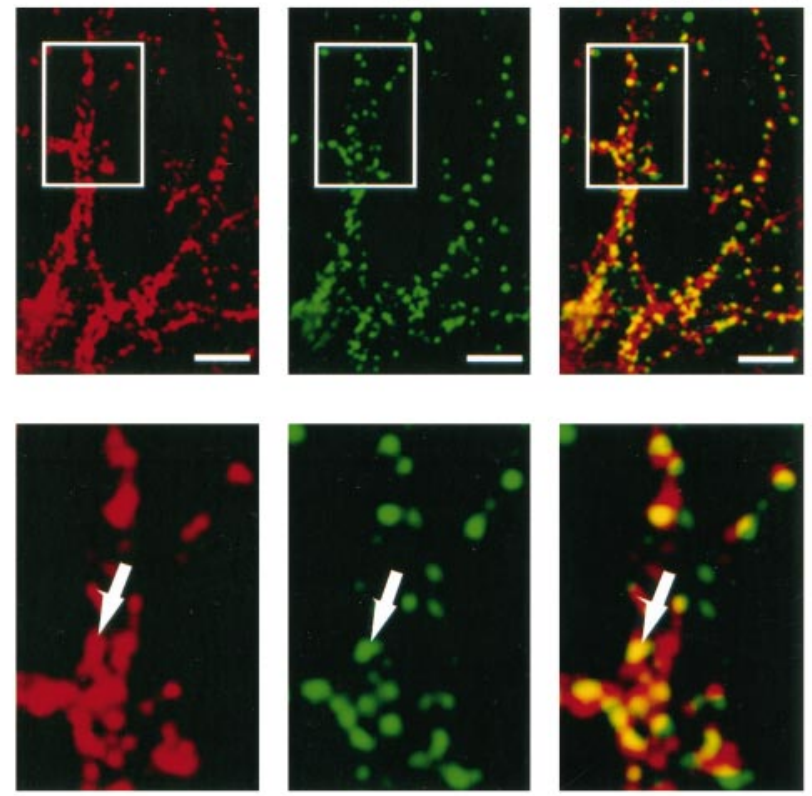

C

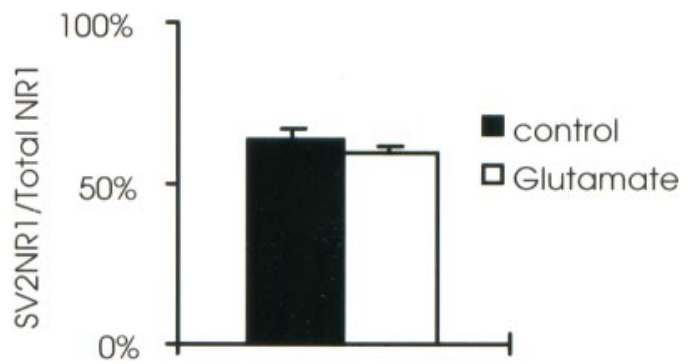

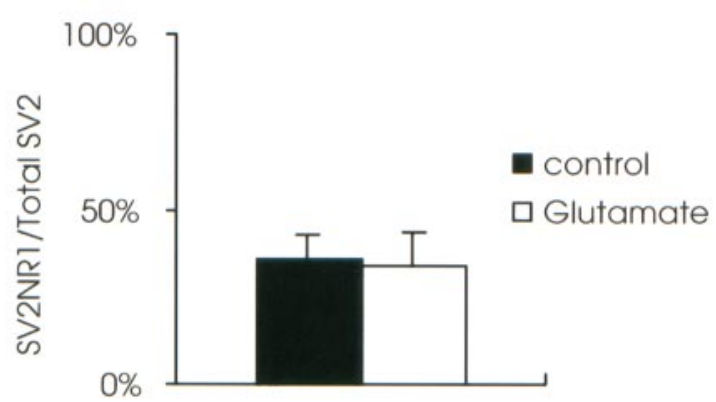

Figure 2. NR1 subunits do not undergo detectable redistribution under the same conditions. The same experiments as described in Figure 1 were conducted using anti-NR1 immmunocytochemical staining in control (untreated) $(A)$ and glutamate-treated $(B)$ neurons. Scale bars, $5 \mu \mathrm{m}$. Arrows in $A$ and $B$ point out examples of an NR1 puncta colocalized with SV2. Analysis of the proportion of NR1-positive puncta associated with synapses ( $C$ ) and the number of synapses associated with detectable concentration of NR1 immunoreactivity $(D)$ were determined as in Figure 1.

brane depolarization caused by activation of AMPA receptors, we examined the effects on GluR1 distribution of replacing media with high potassium (150 mM) solution for $30 \mathrm{~min}$. This manipulation failed to cause any apparent redistribution of GluR1 subunits away from synapses. Rapid redistribution of GluR1containing receptors was still observed, however, when AMPA or glutamate was applied to cultures together with high potassium.

Because a major consequence of depolarizing neurons is to elicit a rise in postsynaptic $\mathrm{Ca}^{2+}$, it also was of interest to determine whether such an increase in intracellular $\mathrm{Ca}^{2+}$ level is required for the redistribution of GluR1 caused by application of AMPA. To prevent AMPA-induced changes in intracellular $\mathrm{Ca}^{2+}$, we applied the membrane-permeable $\mathrm{Ca}^{2+}$ chelator BAPTA-AM $(10 \mu \mathrm{M})$ for $1 \mathrm{hr}$ before AMPA application. Although we cannot rule out some effect of BAPTA-AM, significant redistribution of GluR1 was still observed in cells incubated under these conditions (data not shown). Furthermore, AMPA- induced redistribution of GluR1 was not blocked by $1 \mu \mathrm{M}$ tetrodotoxin (data not shown). Together, the results thus far suggest that the rapid redistribution of GluR1 subunits is mediated specifically by ligand-induced activation of AMPA receptors.

It is well known that glutamate can cause significant excitotoxicity both in vivo and in cultured neurons (Choi, 1994). Previous studies of cultured hippocampal neurons indicate that concentrations of glutamate comparable to those used in the present studies are capable of causing irreversible cell damage (Michaels and Rothman, 1990; Schinder et al., 1996). To evaluate the possibility that the observed redistribution of GluR1 subunits is a consequence of cell death, neuronal viability was determined using the established method of trypan blue staining (Schinder et al., 1996) $24 \mathrm{hr}$ after incubation of cultures with glutamate or AMPA under conditions identical to those used for studies of receptor localization. Application of glutamate for $15 \mathrm{~min}$ caused excitotoxicity that was in significant excess to that observed in untreated neu- 


\section{A. Control}

GluR1
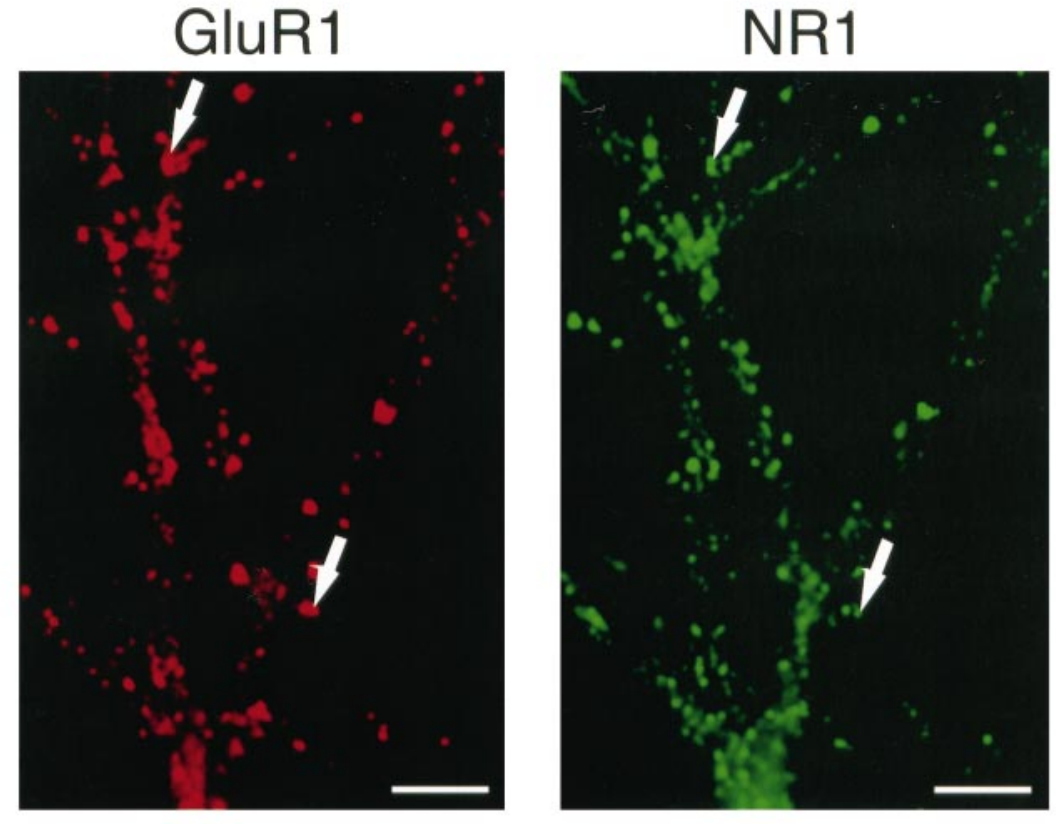

Superimposed

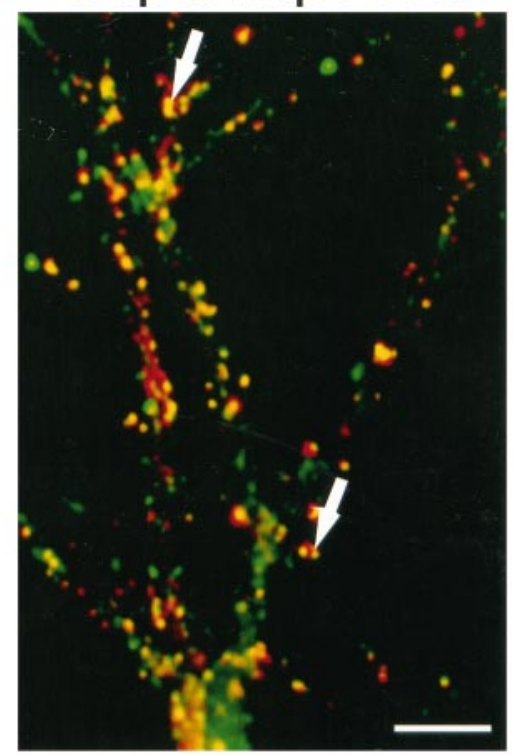

\section{B. Glutamate}

\section{GluR1}

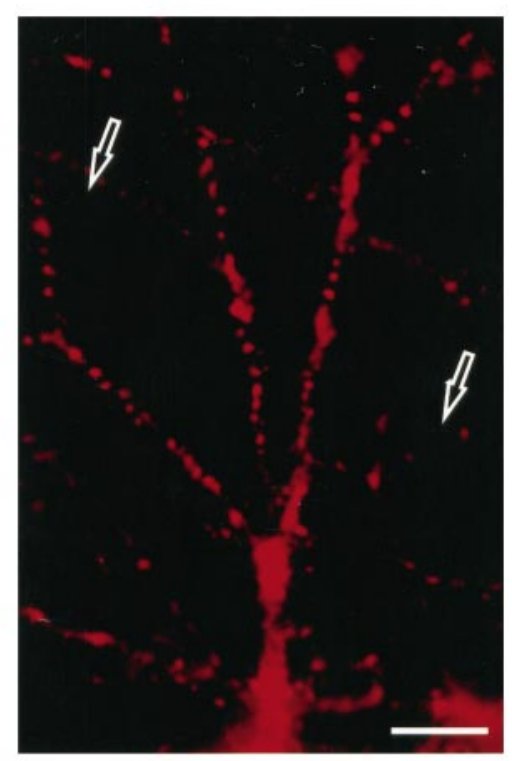

\section{NR1}

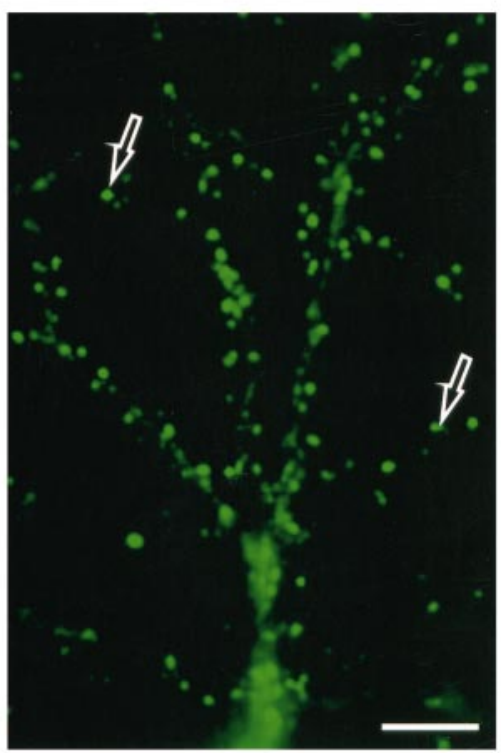

\section{Superimposed}

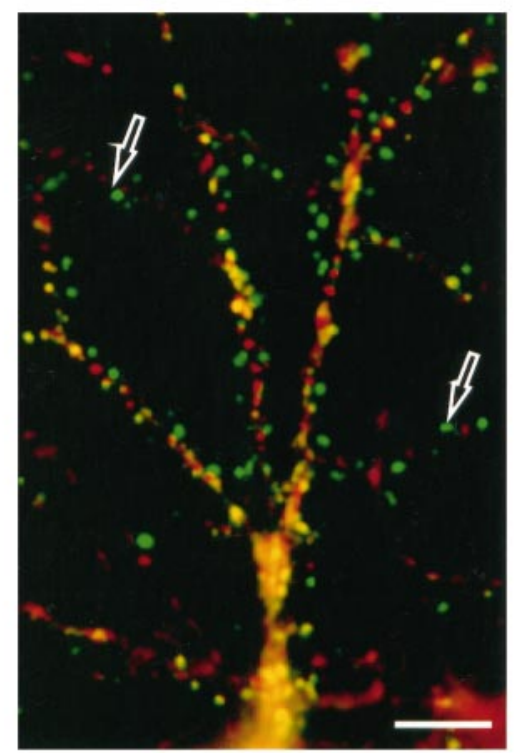

Figure 3. Dual localization of GluR1 and NR1 subunits in the same neurons. Cultured hippocampal neurons were incubated in the absence $(A)$ or presence $(B)$ of $100 \mu \mathrm{M}$ glutamate for $15 \mathrm{~min}$ and then fixed, permeabilized, and processed for immunocytochemical analysis using rabbit anti-GluR1 and mouse anti-NR1, as described in Materials and Methods. Representative fluorescence micrographs obtained under each condition are displayed. Arrows in $A$ indicate examples of puncta associated with both GluR1 and NR1 immunoreactivity. Open arrows in $B$ indicate examples of NR1-containing puncta devoid of any detectable GluR1 immunoreactivity, which were observed frequently in glutamate-treated cultures. Scale bars, $5 \mu \mathrm{m}$.

rons. However, consistent with previous studies establishing a requirement for NMDA receptor activation in rapid excitotoxicity (Michaels and Rothman, 1990), application of AMPA caused significantly less excitotoxicity (Fig. $5 A$ ). This pronounced difference in the excitotoxic actions of AMPA versus glutamate appli- cation contrasts markedly with their similar effects on the redistribution of GluR1 (Fig. 4). In addition, the rapid redistribution of GluR1 subunits observed in AMPA-treated neurons appeared to be reversed after washout of AMPA (Fig. 5B), suggesting that the rapid redistribution of GluR1 subunits is reversible. Thus, we 
Figure 4. Rapid redistribution of GluR1 subunits is induced by AMPA and does not require activation of NMDA receptors. $A$, In control (untreated) neurons, the majority of GluR1 receptor clusters were localized at the periphery of dendritic shafts, consistent with synaptic localization. $B$, Within 15 min after the addition of $100 \mu \mathrm{M}$ AMPA to the culture medium, a pronounced redistribution of GluR1 subunits was observed, which was qualitatively identical to that induced by glutamate. Note that most punta are in the center of the dendritic shaft. $C$, Coapplication of the NMDA receptor antagonist APV $(100 \mu \mathrm{M})$ together with AMPA did not block the AMPA-induced redistribution of GluR1 subunits, confirming that this redistribution does not require activation of NMDA receptors. Scale bars, $5 \mu \mathrm{m}$.
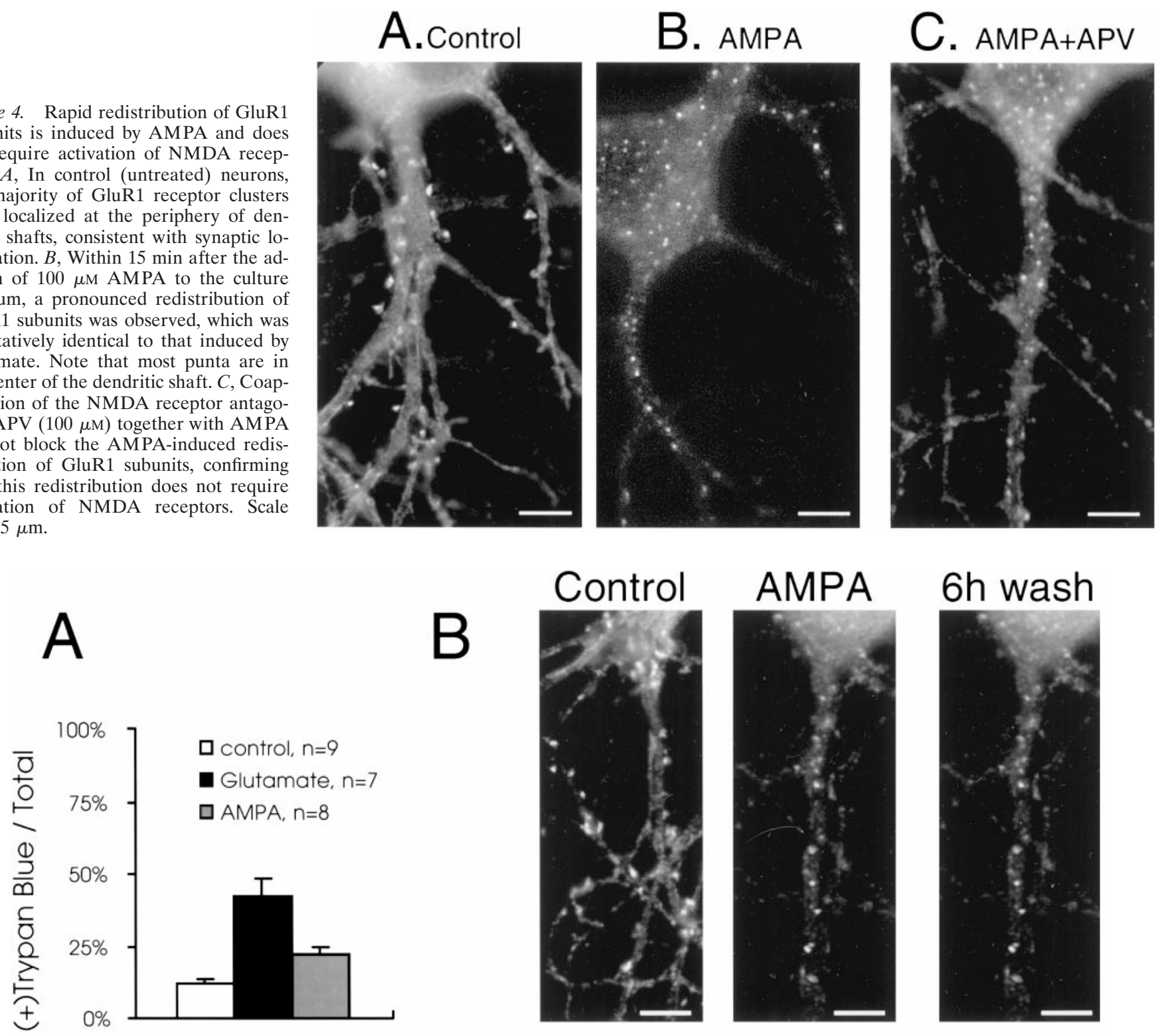

Figure 5. Redistribution of GluR1 subunits can be dissociated from ligand-induced neurotoxicity and is reversible after removal of agonist. $A$, Neurotoxicity in cultured hippocampal neurons was assayed by trypan blue exclusion $24 \mathrm{hr}$ after treatment of cultures for 15 min with $100 \mu \mathrm{M}$ glutamate or AMPA under conditions identical to those used to induce rapid redistribution of GluR1 subunits. Whereas glutamate caused substantial neurotoxicity, AMPA caused significantly less neurotoxicity [although still significantly more than observed in control (untreated) cultures]. B, AMPA-induced redistribution of GluR1 subunits was reversed within $6 \mathrm{hr}$ after washout of AMPA. Scale bars, $5 \mu \mathrm{m}$.

conclude that the rapid redistribution of GluR1 observed in the present study represents a specific regulation of the composition of postsynaptic sites that is not the result of agonist-induced excitotoxicity.

Because of its reduced potential to cause neurotoxicity, AMPA was used to induce GluR1 redistribution for more detailed studies of this process. GluR1-containing receptors present in control neurons were associated with a large number of structures resembling dendritic spines, as indicated by their localization at the periphery of the dendritic shaft and colocalization with intense staining with phalloidin (Fig. 6A, arrow, example of a presumed dendritic spine with colocalized GluR1 immunoreactivity), which labels F-actin concentrated in these structures (Allison et al., 1998). In cells treated with AMPA, however, phalloidin-positive spinous structures were observed that were not associated with detectable GluR1 immunoreactivity (Fig. 6B, green, arrow). Furthermore, numerous GluR1 puncta were visualized in AMPAtreated neurons that were not associated with phalloidin-positive structures and were located toward the base of the dendritic shaft (Fig. 6B, red) or in the cell body (Fig. 4B). These observations suggest that AMPA caused a rapid and specific redistribution of GluR1-containing receptors away from dendritic spines, although phalloidin-positive structures were observed both in untreated and AMPA-treated neurons.

We further examined the localization of GluR1 in dendritic processes at higher resolution using laser scanning confocal microscopy. Because of the complex morphology and small size of these processes, only a small number of receptor clusters were imaged in an individual optical section. Therefore, threedimensional image reconstruction was used to analyze the local- 


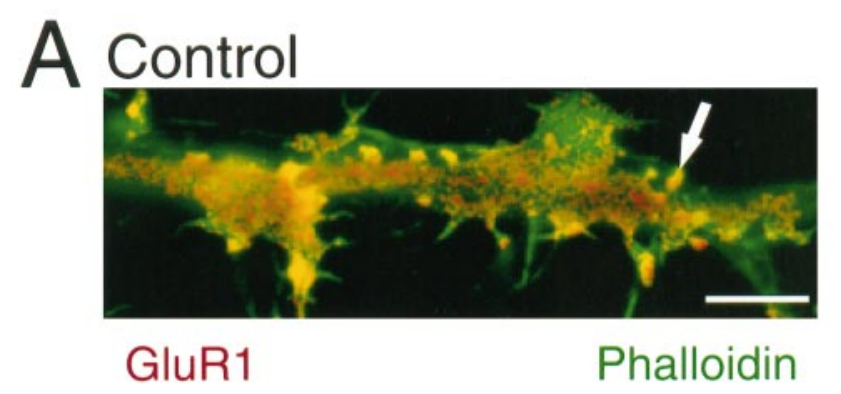

GluR1
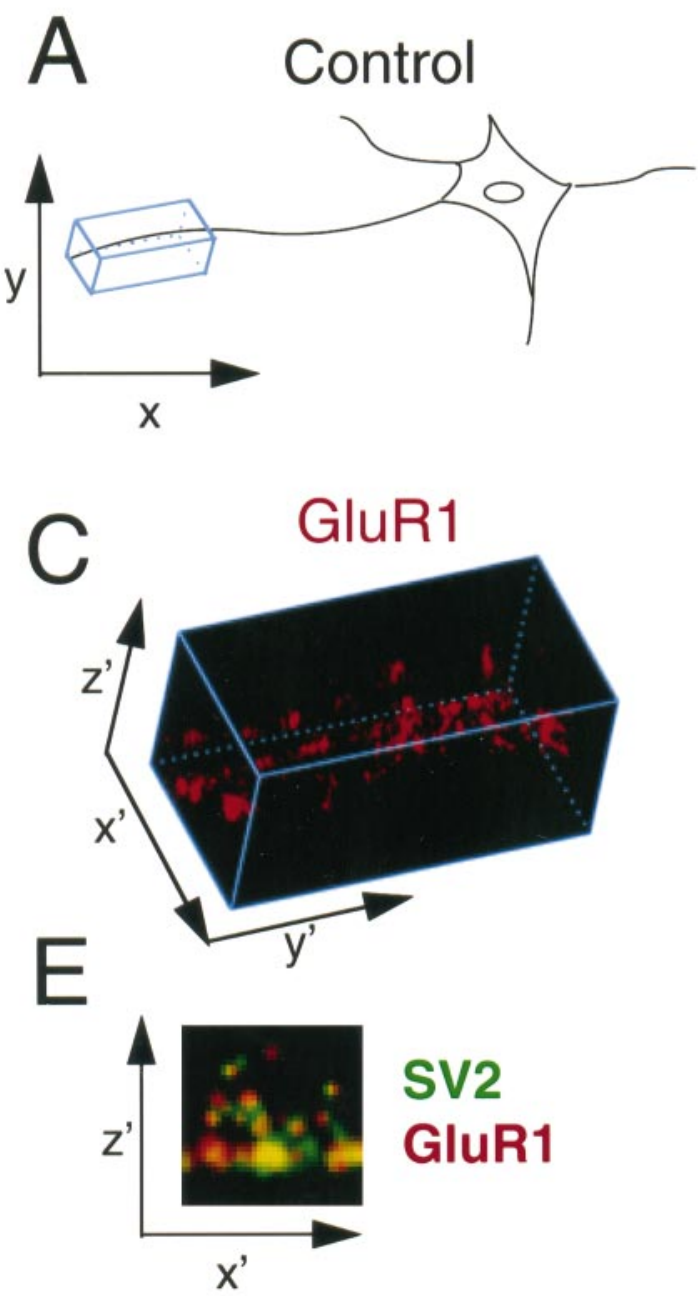
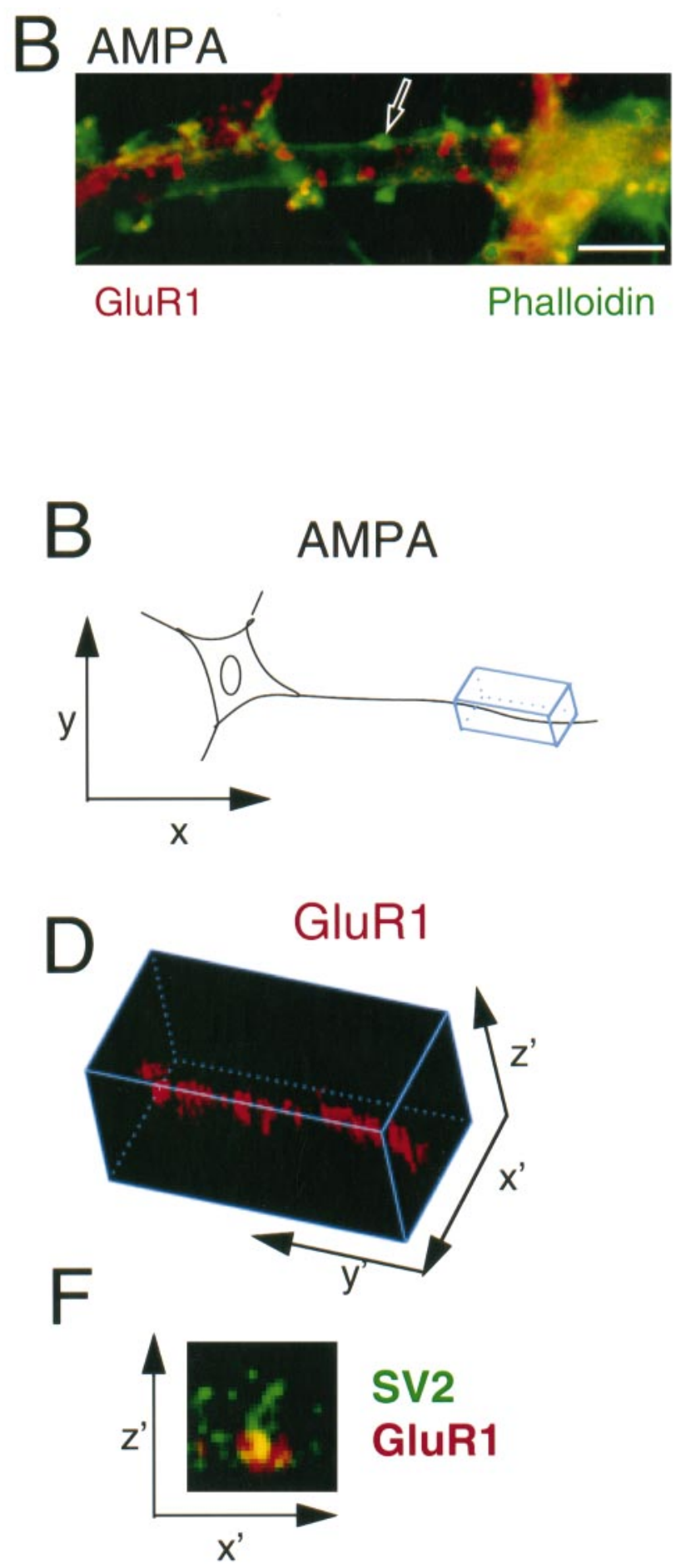

Figure 6. Top. Rapid, AMPA-induced redistribution of GluR1 subunits from dendritic spines. $A$, In control neurons, many clusters of GluR1 subunits (red) colocalized with phalloidin staining ( green), consistent with colocalization in dendritic spines. Arrow indicates an example of such a structure, which appears yellow in the merged image. $B$, After incubation of cultures with $100 \mu \mathrm{M}$ AMPA for 15 min, many GluR1-containing puncta were observed to be distinct from phalloidin-stained extensions of the dendritic membrane, consistent with a redistribution of GluR1 subunits away from dendritic spines. Arrow indicates an example of such a phalloidin-positive dendritic specialization, observed frequently in AMPA-treated neurons, which was devoid of detectable GluR1 immunoreactivity. Scale bars, $2 \mu \mathrm{m}$.

Figure 7. Bottom. Analysis of AMPA-induced redistribution of GluR1 subunits in individual dendrites using confocal fluorescence microscopy and three-dimensional image reconstruction. Schematic view of a control neuron $(A)$ and an AMPA-treated neuron $(B)$, showing the scanned region containing an individual dendritic shaft (box). $C, D$, Representative three-dimensional reconstructions displaying the localization of GluR1 immunoreactivity in dendritic specializations viewed obliquely (as indicated by the schematic) using the maximum projection technique in a control (untreated) neuron and an AMPA-treated (100 $\mu \mathrm{M}$ for $15 \mathrm{~min})$ neuron, respectively. $E, F$, Collapsed end-on images of the same three-dimensional image reconstructions as in $C$ and $D$, displayed using the maximum projection technique along the $z^{\prime}$-axis, emphasizing the AMPA-induced redistribution of GluR1 subunits (red) away from SV2-positive synaptic sites (green) located at the periphery. 


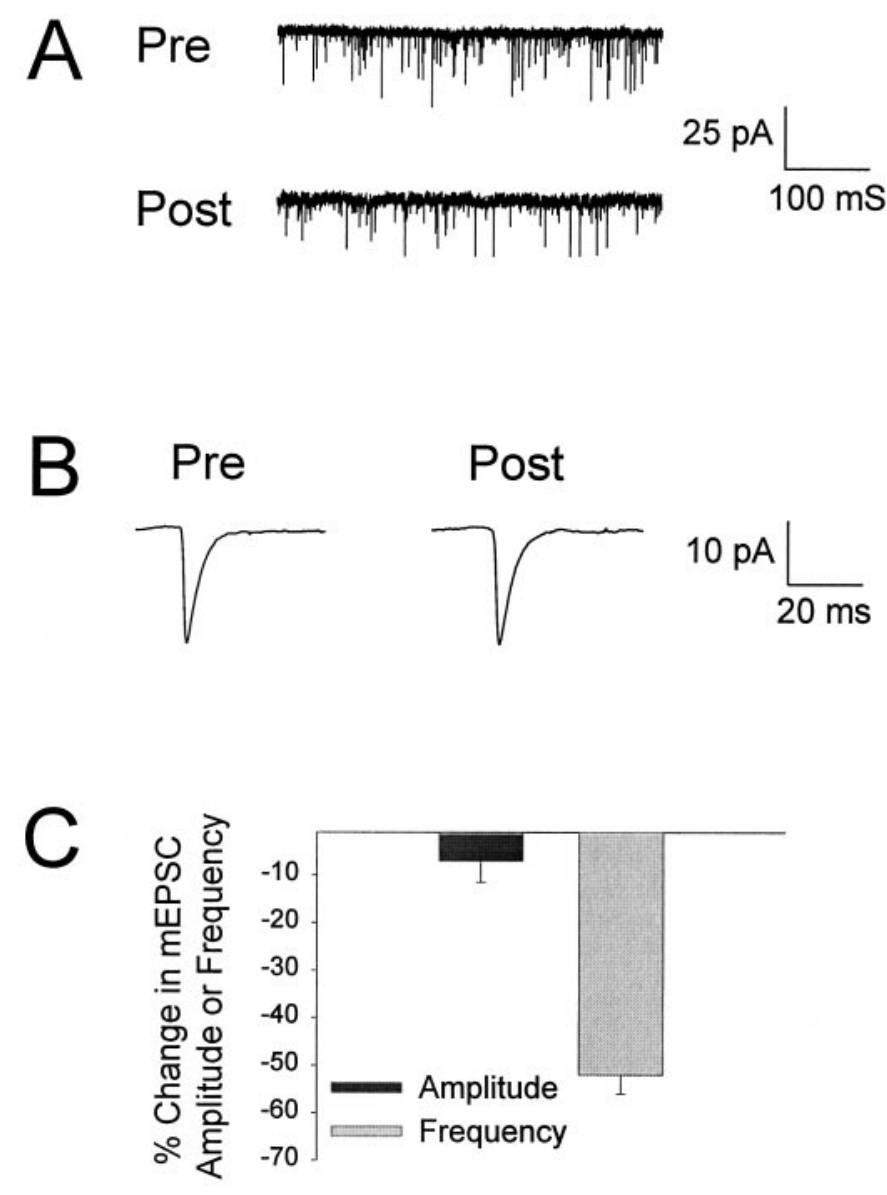

Figure 8. The rapid redistribution of GluR1 subunits is associated with decreased frequency of mEPSCs. $A$, Traces of miniature synaptic events recorded from cultured hippocampal neurons in the presence of lidocaine $(10 \mu \mathrm{M})$ before (Pre) and after (Post) application of $100 \mu \mathrm{M}$ AMPA to cells for $15 \mathrm{~min}$, followed by rapid washout. $B$, Averaged mEPSC (250 events) pretreatment and posttreatment with AMPA from experiment in $A$ show only a slight reduction in amplitude. $C$, Summary of the effects of AMPA treatment on the mean amplitude and frequency of mEPSCs. Error bars represent SEM ( $n=9$ cells).

ization of GluR1 and SV2 immunoreactivity in serial optical sections imaged at maximum resolution by scanning dendritic processes from control and AMPA-treated neurons (Fig. 7 $A, B$ ). A representative three-dimensional reconstruction of GluR1 immunoreactivity in a portion of a dendritic process from an untreated neuron is shown in Figure $7 C$, and an equivalent reconstruction of a process from an AMPA-treated neuron is shown in Figure $7 D$. The AMPA-induced redistribution of GluR1 from peripheral processes to the base or interior of the dendritic process is emphasized by comparing Figure $7, E$ and $F$, which represent a collapsed end-on $\left(x^{\prime} / z^{\prime}\right)$ view down the cross section of reconstructed processes from untreated and AMPA-treated neurons, respectively. GluR1 and SV2 colocalize extensively at the periphery of dendritic processes (Fig. $7 E$, yellow), whereas a centripetal redistribution of GluR1 immunoreactivity away from the peripheral synaptic sites (marked by SV2 immunoreactivity) was observed in dendritic processes from AMPA-treated neurons (Fig. $7 F$, red and green, respectively).

The rapid redistribution of GluR1 subunits away from the synapse would be expected to have significant effects on synaptic transmission. To examine the functional consequences of the rapid redistribution of GluR1-containing receptors, synaptic transmission in cultured neurons was analyzed by recording mEPSCs before and after bath application of AMPA. As expected, the cell membrane potential depolarized from approximately -60 to $0 \mathrm{mV}$ after the application of AMPA and returned to original levels when the drug was washed out. Comparison of whole-cell recordings before and after treatment showed that application of AMPA caused a large decrease in the frequency of mEPSCs without causing a significant decrease in their mean amplitude (Fig. 8). This result is consistent with the hypothesis that the rapid redistribution of GluR1 subunits influences the efficacy of synaptic transmission in a subset of synaptic sites.

\section{DISCUSSION}

We have demonstrated a rapid, activation-induced redistribution of GluR1-containing receptors from a subset of synaptic sites. This regulatory process appears to be highly specific for GluR1containing AMPA receptors relative to NR1-containing NMDA receptors and occurs without a gross change in neuronal cytoarchitecture, as indicated by the apparent preservation of synaptic sites in agonist-treated cells. This redistribution of GluR1 subunits occurs much more rapidly than the previously reported processes of activity-dependent redistribution of AMPA and NMDA receptors, which require several days to be detected experimentally (Rao and Craig, 1997; Lissin et al., 1998). Furthermore, in contrast to these previously reported changes in the subcellular distribution of glutamate receptor subunits, the rapid redistribution of GluR1 observed in the present study does not require activation of NMDA receptors. Thus, we have identified a novel mechanism of receptor regulation, which can rapidly and specifically control the number of GluR1-containing receptors present at synapses.

The observation that this redistribution of GluR1 subunits is induced specifically by AMPA receptor activation, is not induced by depolarization or NMDA receptor-mediated neuronal activity, and is not blocked by the $\mathrm{Ca}^{2+}$ chelator BAPTA-AM suggests the intriguing possibility that the molecular machinery mediating this rapid regulation may be controlled directly by an activated conformation of the AMPA receptor complex itself. In this case, regulated protein interactions with individual receptor subunits located at synaptic sites may play a critical role in mediating the selectivity of subunit redistribution, perhaps independently of downstream signal transduction. Thus, the regulation of ionotropic glutamate receptors may share unanticipated similarities with other classes of receptor protein, such as G-protein-coupled receptors, which are regulated by conformation-dependent interaction of receptors with cytoplasmic regulatory proteins (such as receptor kinases and arrestins) that can occur in the absence of downstream (G-proteinlinked) signal transduction (Freedman and Lefkowitz, 1996; Grady et al., 1997).

Rapid, activation-induced regulation of G-protein-coupled receptors is often associated with lateral redistribution of receptors in the plasma membrane (von Zastrow and Kobilka, 1994; Goodman et al., 1996), followed by rapid endocytosis (Freedman and Lefkowitz, 1996; Okamoto et al., 1998). Although these mechanisms obviously regulate the subcellular localization of receptors, they are also thought to play a fundamental role in modulating the efficacy of signal transduction to distinct effectors (Vieira et al., 1996; Grady et al., 1997). To our knowledge, the present results provide the first direct evidence that a similar process of receptor redistribution may regulate ionotropic glutamate receptors. Although the precise mechanisms mediating glutamate re- 
ceptor redistribution remain to be elucidated, the present results indicate that GluR1-containing and NR1-containing receptors differ greatly in their ability to undergo rapid redistribution in neurons, although these receptors are frequently concentrated in the same postsynaptic sites. This selectivity of regulation may be conferred by the highly specific interaction of AMPA and NMDA receptors with distinct cytoplasmic proteins associated with postsynaptic densities (Kornau et al., 1995, 1997; Dong et al., 1997; Srivastava et al., 1998). Indeed, previous studies indicate that AMPA receptors can be dissociated from the neuronal cytoskeleton much more readily than NMDA receptors (Allison et al., 1998).

The ligand-induced removal of GluR1-containing receptors from the synaptic plasma membrane would be expected to depress synaptic transmission. Consistent with this prediction, prolonged agonist exposure caused a reduction in the frequency of mEPSCs. Thus, we believe that we have identified a heretofore unknown mechanism for the functional regulation of synaptic AMPA receptors. For example, it is possible that the rapid and reversible redistribution of GluR1-containing AMPA receptors observed in the present studies may play a role in the physiological phenomenon of silent synapses in which the efficacy of individual synapses is regulated by a dramatic change in the number of functional AMPA-type receptors present in the postsynaptic membrane (Isaac et al., 1995; Liao et al., 1995; Durand et al., 1996). However, because multiple mechanisms contribute to the physiological regulation of signal transduction in the postsynaptic plasma membrane, the precise role of this specific mechanism of receptor regulation remains to be determined. Furthermore, because the experimental conditions used to induce the rapid redistribution of GluR1-containing receptors are not achieved under normal physiological conditions (i.e., bath application of agonist), it remains to be determined whether rapid redistribution of AMPA receptor subunits is induced by physiological synaptic activity.

Our immunocytochemical and electrophysiological data are consistent with the hypothesis that AMPA causes an "all-ornone" removal of AMPA receptors from a subset of synaptic sites. In this regard, the rapid regulation of GluR1 receptors may differ significantly from rapid, ligand-induced redistribution of certain G-protein-coupled receptors, which is characterized by a partial decrease in the number of surface receptors observed at steady state attributable to continuous endocytosis and recycling in the presence of agonist. However, caution is required in interpreting the present data because of the limited sensitivity of our analyses. Indeed, it is unlikely that our immunochemical methods are sufficiently sensitive to detect receptor subunits in synapses that contain very small (but perhaps functionally sufficient) amounts of receptor protein. Similarly, we cannot state with certainty whether the decrease in mEPSC frequency observed in the present study represents complete "silencing" of a restricted subset of synapses or a graded reduction in a larger number of synapses, leading to a limited subset of synapses falling below our threshold for detection by whole-cell electrophysiology. Nevertheless, our observations that the redistribution of AMPA receptors is associated with a relatively small change in mEPSC amplitude compared with the more pronounced effect on frequency does suggest some heterogeneity in the response of individual synapses to activation. Further studies will be necessary to elucidate specific mechanisms underlying the observed redistribution of synaptic glutamate receptors and to determine their contribution to graded versus all-or-none regulation of synaptic efficacy.

The experimental conditions used to elicit the rapid redistribution of AMPA receptors are similar to those used to induce excitotoxicity in hippocampal neurons. Importantly, however, the rapid redistribution of GluR1 could be induced under conditions that caused relatively little neuronal death (i.e., AMPA stimulation in the presence of APV). Furthermore, this redistribution of receptor subunits was reversible after removal of agonist. Thus, the rapid, activation-induced redistribution of GluR1-containing receptors observed in the present study is unlikely to be a direct consequence of excitotoxicity. It is possible, however, that rapid redistribution of GluR1-containing AMPA receptors functions physiologically in the context of excitotoxicity, perhaps as a mechanism to protect neurons from excessive stimulation by high concentrations of glutamate. If this is true, elucidating mechanisms that mediate the rapid redistribution of receptor components from the synaptic plasma membrane may be of fundamental importance to understanding mechanisms of neural injury and may therefore define novel therapeutic targets for the development of neuroprotective agents.

In conclusion, we have identified a novel type of regulation of the subcellular localization of ionotropic glutamate receptors, which differs substantially from previously described mechanisms of glutamate receptor regulation. These observations establish that receptor trafficking mechanisms mediate rapid regulation of ionotropic glutamate receptors, and they suggest that these mechanisms of receptor regulation may play an important role in modulating postsynaptic responses to physiological or pathophysiological stimuli.

\section{REFERENCES}

Allison DW, Gelfand VI, Spector I, Craig AM (1998) Role of actin in anchoring postsynaptic receptors in cultured hippocampal neurons: differential attachment of NMDA versus AMPA receptors. J Neurosci 18:2423-2436.

Brewer GJ, Torricelli JR, Evege EK, Price PJ (1993) Optimized survival of hippocampal neurons in B27-supplemented Neurobasal, a new serum-free medium combination. J Neurosci Res 35:567-576.

Choi DW (1994) Calcium and excitotoxic neuronal injury. Ann NY Acad Sci 747:162-171.

Craig AM, Blackstone CD, Huganir RL, Banker G (1993) The distribution of glutamate receptors in cultured rat hippocampal neurons: postsynaptic clustering of AMPA-selective subunits. Neuron 10:1055-1068.

Dong H, O’Brien RJ, Fung ET, Lanahan AA, Worley PF, Huganir RL (1997) GRIP: a synaptic PDZ domain-containing protein that interacts with AMPA receptors. Nature 386:279-284.

Durand GM, Kovalchuk Y, Konnerth A (1996) Long-term potentiation and functional synapse induction in developing hippocampus. Nature 381:71-75.

Freedman NJ, Lefkowitz RJ (1996) Desensitization of G proteincoupled receptors. Recent Prog Horm Res 51:319-351.

Goodman OJ, Krupnick JG, Santini F, Gurevich VV, Penn RB, Gagnon AW, Keen JH, Benovic JL (1996) Beta-arrestin acts as a clathrin adaptor in endocytosis of the beta2-adrenergic receptor. Nature 383:447-450.

Grady EF, Bohm SK, Bunnett NW (1997) Turning off the signal: mechanisms that attenuate signaling by $\mathrm{G}$ protein-coupled receptors. Am J Physiol 273:G586-G601.

Hausdorff WP, Caron MG, Lefkowitz RJ (1990) Turning off the signal: desensitization of beta-adrenergic receptor function. FASEB J 4:2881-2889.

Isaac JT, Nicoll RA, Malenka RC (1995) Evidence for silent synapses: implications for the expression of LTP. Neuron 15:427-434.

Kornau HC, Schenker LT, Kennedy MB, Seeburg PH (1995) Domain interaction between NMDA receptor subunits and the postsynaptic density protein PSD-95. Science 269:1737-1740. 
Kornau HC, Seeburg PH, Kennedy MB (1997) Interaction of ion channels and receptors with PDZ domain proteins. Curr Opin Neurobiol 7:368-373.

Lester RA, Quarum ML, Parker JD, Weber E, Jahr CE (1989) Interaction of 6-cyano-7-nitroquinoxaline-2,3-dione with the $N$-methyl-Daspartate receptor-associated glycine binding site. Mol Pharmacol 35:565-570.

Liao D, Hessler NA, Malinow R (1995) Activation of postsynaptically silent synapses during pairing-induced LTP in CA1 region of hippocampal slice. Nature 375:400-404.

Lissin DV, Gomperts SN, Carroll RC, Christine CC, Kalman D, Kitamura M, Hardy S, Nicoll RA, Malenka RC, von Zastrow M (1998) Activity differentially regulates the surface expression of synaptic AMPA and NMDA glutamate receptors. Proc Natl Acad Sci USA 12:7097-7102.

Michaels RL, Rothman SM (1990) Glutamate neurotoxicity in vitro: antagonist pharmacology and intracellular calcium concentrations. J Neurosci 10:283-292.

O'Brien RJ, Lau LF, Huganir RL (1998) Molecular mechanisms of glutamate receptor clustering at excitatory synapses. Curr Opin Neurobiol 8:364-369.

Okamoto T, Schlegel A, Scherer PE, Lisanti MP (1998) Caveolins, a family of scaffolding proteins for organizing "preassembled signaling complexes" at the plasma membrane. J Biol Chem 273:5419-5422.

Rao A, Craig AM (1997) Activity regulates the synaptic localization of the NMDA receptor in hippocampal neurons. Neuron 19:801-812.

Schinder AF, Olson EC, Spitzer NC, Montal M (1996) Mitochondrial dysfunction is a primary event in glutamate neurotoxicity. J Neurosci 16:6125-6133.

Sheng M (1997) Excitatory synapses. Glutamate receptors put in their place. Nature 386:221-223.

Srivastava S, Osten P, Vilim FS, Khatri L, Inman G, States B, Daly C, DeSouza S, Abagyan R, Valtschanoff JG, Weinberg RJ, Ziff EB (1998) Novel anchorage of GluR2/3 to the postsynaptic density by the AMPA receptor-binding protein ABP. Neuron 21:581-591.

Turrigiano GG, Leslie KR, Desai NS, Rutherford LC, Nelson SB (1998) Activity-dependent scaling of quantal amplitude in neocortical neurons. Nature 391:892-896.

Vieira AV, Lamaze C, Schmid SL (1996) Control of EGF receptor signaling by clathrin-mediated endocytosis. Science 274:2086-2089.

von Zastrow M, Kobilka BK (1994) Antagonist-dependent and -independent steps in the mechanism of adrenergic receptor internalization. J Biol Chem 269:18448-18452. 